\title{
A Debate on Safety of the Irradiated Food
}

\author{
R. Sundaralingam \\ Assistant Professor, Department of Microbiology, \\ Madras Christian College (Autonomous), Tambaram, Chennai, Tamil nadu, India \\ *Corresponding Author: R. Sundaralingam, Assistant Professor, Department of Microbiology, Madras \\ Christian College (Autonomous), Tambaram, Chennai, Tamil nadu, India
}

After many years of research and development in food safety, more than 60 countries worldwide have regulations allowing the use of irradiation for one or more food products. Irradiation is nothing new, but then neither is the debate about it.

Irradiation technique has been used to sterilize medical and hospital supplies, food packaging materials and cosmetics ingredients for years, but in maintaining food safety this is a novel technique.

Irradiation is a process in which food is exposed to high doses of radiation in the form of gamma rays, $\mathrm{X}$-rays or electron beams. Irradiation is claimed to kill bacteria in food, both good and bad, but has no effect on the infectious agent that causes mad cow disease, or on viruses, such as those that cause hepatitis. The process produces a similar effect to pasteurization, cooking or other forms of heat treatment, but with less effect on look and texture. Irradiated food has been exposed to radioactivity but does not become radioactive itself.

Radiation processing of food involves controlled application of the energy of short wave length radiations of the electromagnetic spectrum, which includes radiowaves, microwaves, infrared, visible and ultraviolet light. These short wave length radiations are also known as ionizing radiations and include gamma rays, accelerated electrons, and X-rays.

Food is irradiated to extend its shelf life and kill pests like fruit flies. It uses gamma rays with short wavelengths and high frequencies that penetrate food so rapidly that little or no heat is produced. Microwaving, which uses longer wavelengths, causes foods to heat rapidly.

Currently, the US Food and Drug Administration has approved irradiation of foods including meat and poultry, shell eggs, fruits and vegetables, herbs, spices and flour. However, only a few of these approved foods are actually produced commercially; currently, irradiated foods are limited to small amounts of ground beef, spices, and some imported fruit such as papayas. Irradiated foods sold in grocery stores are required to be labeled.

\section{Benefits of Irradiated Foods}

Radiation processing of food can achieve different objectives in different foods. Some of the major benefits of radiation processing of food are:

- Insect disinfestation of dried stored products

- Phytosanitation to overcome quarantine barriers in fruits and vegetables.

- Inhibition of sprouting in tubers, bulbs and rhizomes.

- Delay in ripening and senescence of fruits

- Enhancement in shelf -life by destruction of spoilage microbes

- Elimination of pathogens and parasites

\section{What's wrong with irradiation?}

The most important issue raised by consumer groups, the mass media, and even a few governmental representatives is the safety of irradiated food. It covers a wide range of technical subjects, including 
free radicals, radiolytic products, mutagenic and carcinogenic substances, polyploidy, vitamin losses, dangerous bacteria, and toxins.

Irradiation damages food by breaking up molecules and creating free radicals. The free radicals kill some bacteria, but they also bounce around in the food, damage vitamins and enzymes, and combine with existing chemicals (like pesticides) in the food to form new chemicals, called unique radiolytic products (URPs).

Some of these URPs are known toxins (benzene, formaldehyde, lipid peroxides) and some are unique to irradiated foods.

Scientists have not studied the long-term effect of these new chemicals in our diet. Therefore, we cannot assume they are safe.

Irradiated foods can lose 5\%-80\% of many vitamins (A, C, E, K and B complex). The amount of loss depends on the dose of irradiation and the length of storage time.

Most of the food in the American diet is already approved by the U.S. Food and Drug Administration (FDA) for irradiation: beef, pork, lamb, poultry, wheat, wheat flour, vegetables, fruits, shell eggs, seeds for sprouting, spices, herb teas. (Dairy is already pasteurized). A food industry petition currently before the FDA asks for approval for luncheon meats, salad bar items, sprouts, fresh juices and frozen foods. Another petition before the USDA asks for approval for imported fruits and vegetables.

Irradiation damages the natural digestive enzymes found in raw foods. This means the body has to work harder to digest them. If unlabeled, raw foods that have been irradiated look like fresh foods, but nutritionally they are like cooked foods, with decreased vitamins and enzymes. The FDA allows these foods to be labeled "fresh."

\section{Conclusion - Irradiated foods are Safe}

International groups of scientists have evaluated extensive data from safety studies of irradiated foods and have found no basis for concern. Studies include those analyzing chemical changes in irradiated foods - no matter how minute - and whether they could give rise to long-term human toxicity.

A number of sensitive tests were employed in such studies, including host-mediated assays, cytogenetic analysis, micronucleus tests and long-term multigeneration feeding studies using rats, mice, dogs, monkeys, and other animals. Many of these studies were carried out or co-ordinated by the International Project in the Field of Food Irradiation, based in Karlsruhe, Federal Republic of Germany, from 1971 to 1981 . Data generated from them were evaluated by leading experts in toxicology, microbiology, nutrition, and chemistry appointed by the Food and Agriculture Organization (FAO), IAEA, and World Health Organization (WHO) in 1976 and 1980.

In 1980, a significant amount of new data on animal feeding tests and radiation chemistry were available for evaluation.

On the basis of these data, the Joint Expert Committee on Food Irradiation (JECFI) of the FAO, IAEA, and WHO came to the conclusion that "irradiation of any food commodity up to an overall average dose of 10 kilogray presents no toxicological hazard; hence, toxicological testing of foods so treated is no longer required".

It also found that irradiation up to this dose level "introduces no special nutritional or microbiological problems".

Citation: R. Sundaralingam, "A Debate on Safety of the Irradiated Food ", International Journal of Research studies in Microbiology and Biotechnology, vol. 3, no. 4, p. 4-5, 2017. http://dx.doi.org/10.20431/ 2454-9428.0304002

Copyright: (C) 2017 Authors. This is an open-access article distributed under the terms of the Creative Commons Attribution License, which permits unrestricted use, distribution, and reproduction in any medium, provided the original author and source are credited. 